\title{
Phylogenetic analysis of Helicobacter species based on partial gyrB gene sequences
}

\author{
Correspondence \\ Minna Hannula \\ minna.hannula@helsinki.fi
}

\author{
Minna Hannula and Marja-Liisa Hänninen \\ Department of Food and Environmental Hygiene, Faculty of Veterinary Medicine, University of \\ Helsinki, Helsinki, Finland
}

\section{INTRODUCTION}

The genus Helicobacter belongs to the Epsilonproteobacteria and at the time of writing comprised 23 recognized species (Euzéby, 1997). Helicobacter species infect and colonize the gastrointestinal tracts of a large variety of animal hosts. The most significant Helicobacter species, Helicobacter pylori, has been implicated as an important aetiological factor in a variety of human gastric disorders, such as duodenal and gastric ulcers and gastric cancer (Sanders \& Peura, 2002).

Analysis of 16S rRNA gene sequences is the most common method used for the molecular study of the phylogeny and the taxonomy of bacteria, including Helicobacter species (Dewhirst et al., 2000). However, several examples have indicated that the 16S rRNA gene does not differentiate between certain Helicobacter species, given that intraspecies diversity of $16 \mathrm{~S}$ rRNA genes within some species may be higher than interspecies diversity. Helicobacter bizzozeronii and Helicobacter salomonis, for instance, share a 16S rRNA gene sequence similarity of more than $99 \%$ (Jalava et al., 1997); by contrast, intraspecies diversity within Helicobacter cinaedi and Helicobacter trogontum can reach $4 \%$ (Hänninen et al., 2003; Vandamme et al., 2000). For this reason, other genes such as $h s p 60$ (Mikkonen et al., 2004), the 23S rRNA gene (Dewhirst et al., 2005) and ureB (Hänninen et al., 2005) have been used in phylogenetic studies on Helicobacter, each with particular strengths and weaknesses relating to the amount of sequence information obtained, applicability, labour-intensiveness and distinguishing power. Therefore, it seems evident that no single phylogenetic marker gene alone can clarify the phylogenetic relationships among Helicobacter species but that analyses of several well-established marker genes are needed. This idea was recently supported by Gevers et al. (2005), who discussed the value of multilocus sequence analysis in the phylogenetic analysis of bacteria.

The gyrB gene, which encodes the B-protein subunit of DNA gyrase, has also been utilized in phylogenetic studies. A- and B-proteins are the constituents of the gyrase enzyme, which functions in relieving supercoiling in DNA by creating transient breaks in the double helix (topoisomerase type II). The gyrB gene may be particularly suitable for bacterial identification because it is an evolving, single-copy housekeeping gene that has already proved to be a useful tool in phylogenetic studies of several complex taxa as well as in microarray-based applications (Kasai et al., 2000; Yamamoto \& Harayama, 1995; Soler et al., 2004; Kakinuma et al., 2003).

In the present study the applicability of the gyrB gene for the phylogenetic analysis of Helicobacter species was evaluated, 
and the sequence data obtained from these experiments were employed to develop a PCR-based diagnostic test for the species-specific detection and identification of $H$. bizzozeronii.

\section{METHODS}

Bacterial strains. The partial $\operatorname{gyr} B$ gene was sequenced from 31 strains representing 15 Helicobacter species and one canine isolate of Helicobacter sp. 'flexispira' (Table 1). In addition, the corresponding sequences of $H$. pylori 26695 (=ATCC 700392) and J99 (=ATCC 700824) as well as Campylobacter jejuni NCTC 11168 were obtained from the NCBI nucleotide database.

DNA isolation and PCR amplification. Bacteria were cultured on Brucella blood agar plates (Oxoid) containing selective antimicrobial agents (Hänninen et al., 1996) microaerobically at $37^{\circ} \mathrm{C}$ for 2-3 days. Bacterial mass was collected from two or three plates and genomic DNA was isolated by the method of Pitcher et al. (1989). The preliminary $g y r B$ gene sequence $(1100 \mathrm{bp})$ of all the studied strains was amplified with the degenerate primers UP1 and UP2 $r$ (Yamamoto \& Harayama, 1995). Because the sequencing capacity of automated cycle sequencers is usually less than $700 \mathrm{bp}$, internal primers (HeliF1, HeliF1b, HeliR2, HeliR2b, HeliR2e and HeliR2g), producing DNA fragments of about 600-680 bp, were designed based on these preliminary sequence data, by using a primer design utility (Cybergene $\mathrm{AB}$ ) (Table 2). The internal primers were then used together with specific primers UP1S and UP2Sr (Yamamoto \& Harayama, 1995) so that, for each strain, two DNA fragments with slightly overlapping ends were amplified by PCR (Fig. 1). The number of primers was kept to the minimum by designing primers with broad species specificity (Table 2 ).

PCR was performed in a total volume of $50 \mu \mathrm{l}$ with $2 \mathrm{U}$ Dynazyme polymerase and $5 \mu$ l Dynazyme buffer (Finnzymes), $5 \mathrm{nmol}$ each dNTP, 200 ng purified chromosomal DNA and 20 pmol each primer. The PCR thermal cycling conditions were as follows: denaturation for $1 \mathrm{~min}$ at $94^{\circ} \mathrm{C}$, annealing for $1 \mathrm{~min}$ at $50{ }^{\circ} \mathrm{C}$ and extension for $1 \mathrm{~min}$ at $72{ }^{\circ} \mathrm{C}$, for 30 cycles.

The PCR products were analysed by using $1.5 \%$ agarose gel electrophoresis. PCR products of the expected size were purified

Table 1. Helicobacter strains used in the study

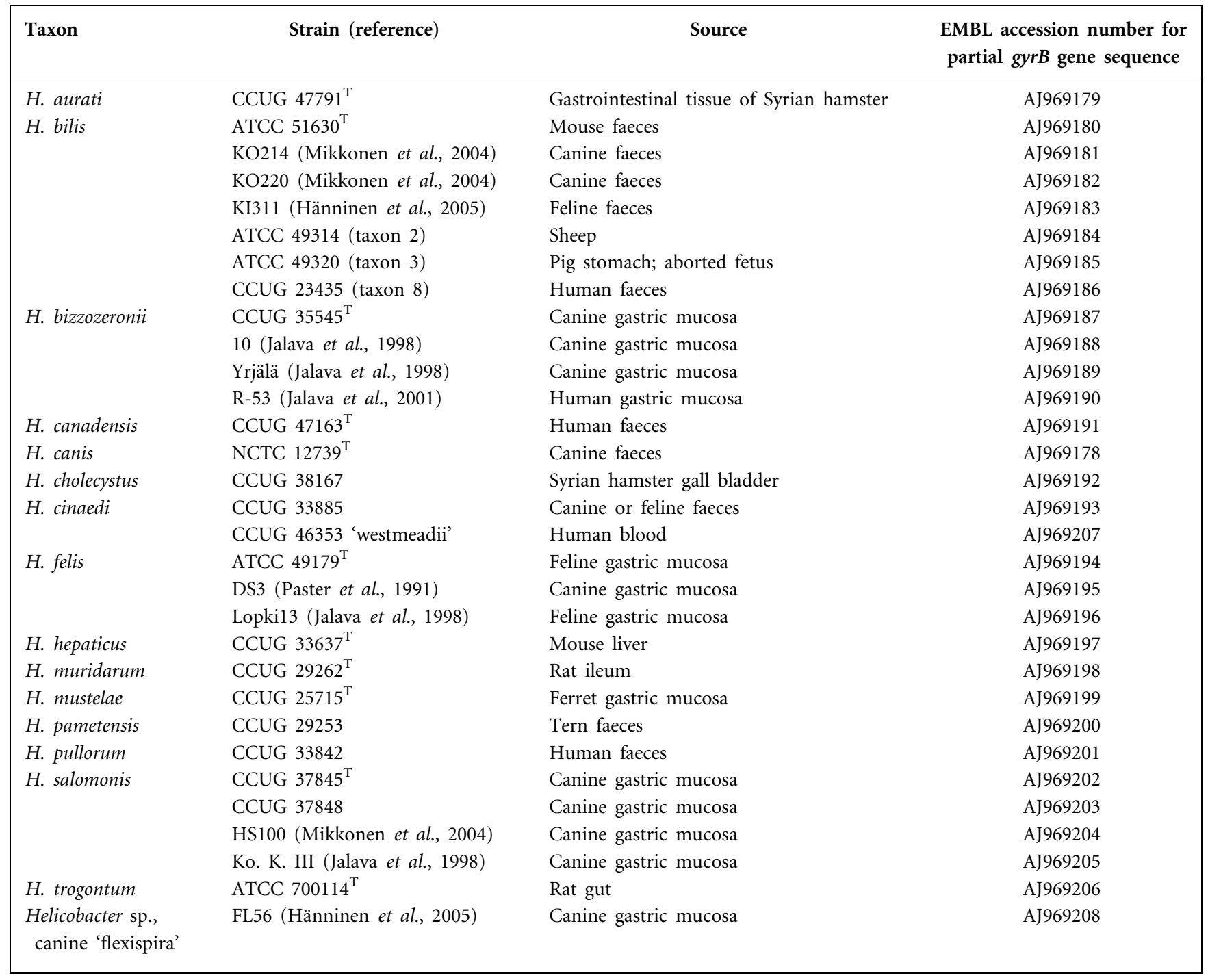


Table 2. Primers used for detection and sequencing of gyrB sequences of Helicobacter species

\begin{tabular}{|c|c|c|c|}
\hline Primer & Direction & Species & Sequence $\left(5^{\prime}-3^{\prime}\right)$ \\
\hline UP2r & Reverse & All & AGCAGGGTACGGATGTGCGAGCCRTCNACRTCNGCRTCNGTCAT \\
\hline $\mathrm{UP} 2 \mathrm{Sr}$ & Reverse & All & AGCAGGGTACGGATGTGCGAGCC \\
\hline HeliF1 & Forward & $\begin{array}{l}\text { H. bilis, FL56, H. trogontum, H. aurati, } \\
\text { H. muridarum, H. cholecystus, } \\
\text { H. pametensis, H. mustelae, H. salomonis, } \\
\text { H. felis, H. bizzozeronii, H. acinonychis }\end{array}$ & GATGGTGGCACGCATGAG \\
\hline HeliF1b & Forward & $\begin{array}{l}\text { H. pullorum, H. canadensis, } H . \text { canis, } \\
\text { H. cinaedi, H. hepaticus }\end{array}$ & CGCACGAAGCAGGATTTAG \\
\hline HeliR2 & Reverse & H. canadensis, H. pullorum, H. bilis, FL56 & CCTAGTTTACCCTTTGTTTG \\
\hline HeliR2b & Reverse & $\begin{array}{l}\text { H. trogontum, H. aurati, H. muridarum, } \\
\text { H. cholecystus, H. mustelae, H. cinaedi, } \\
\text { H. hepaticus }\end{array}$ & ATAATAGCAACAAGCCССTC \\
\hline
\end{tabular}

from $2 \%$ NuSieve GTG low-melting-point agarose gel (BMA) with the Qiaquick gel extraction kit (Qiagen). Nucleotide sequencing was performed by automated cycle sequencing with Big Dye terminators (ABI 377XL; PE Applied Biosystems). Fragments were sequenced at least three times and in both directions to ensure accuracy. With the help of the overlapping ends, the sequence pairs were joined together to form the 1100-bp gyrB gene fragments used in the analysis.

Sequence analysis. The 1100-bp partial gyrB gene sequences obtained in this study and those available in public databases (H. pylori 26695, H. pylori $\mathrm{J} 99$ and C. jejuni NCTC 11168) were aligned using the CLUSTAL $\mathrm{W}$ computer program (Chenna et al., 2003). The ExPASy Translate tool allowed us to translate the nucleotide sequences to the corresponding amino acid sequences. The BioNumerics software program, version 3.5 (Applied Maths), was used in subsequent phylogenetic tree constructions and analysis. The nucleotide and amino acid trees were constructed by using the neighbour-joining method (Saitou \& Nei, 1987). The topology of the trees obtained was evaluated by bootstrap analysis of 1000 replications and by introducing a range of different gap costs. In addition, a maximum-parsimony tree was constructed for comparison.

Species-specific PCR for $\boldsymbol{H}$. bizzozeronii. A specific set of primers for the amplification of the partial $g y r B$ gene sequence of $H$. bizzozeronii was designed on the basis of the acquired $\operatorname{gyr} B$ gene sequences using a primer design utility (Cybergene $\mathrm{AB}$ ). The PCR

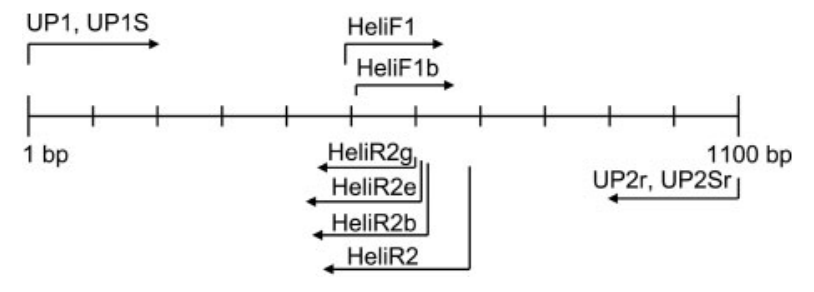

Fig. 1. Sequencing strategy for the $g y r B$ gene fragment of Helicobacter species. thermal cycling conditions were as follows: denaturation for $30 \mathrm{~s}$ at $94{ }^{\circ} \mathrm{C}$, annealing for $30 \mathrm{~s}$ at $55^{\circ} \mathrm{C}$ and extension for $1 \mathrm{~min}$ at $72{ }^{\circ} \mathrm{C}$, for 30 cycles.

The $H$. bizzozeronii-specific primers were GyrbizzoF (5'-GTTGAAATCGCCTTAGCC-3') and GyrbizzoR (5'-GTTCTAAAATCCGTGCTGAG- $\left.3^{\prime}\right)$. The specificity of these primers was tested with the following gastric strains: H. bizzozeronii CCUG $35545^{\mathrm{T}}, 10, \mathrm{R}-53$ and Yrjälä, Helicobacter felis ATCC $49179^{\mathrm{T}}$, DS3 and Lopki13, H. salomonis CCUG 37848 and Ko. K. III, H. pylori 26695 and Helicobacter acinonychis ATCC 51104, with a representative of each of the enterohepatic species listed in Table 1 and with C. jejuni NCTC 11168.

\section{RESULTS AND DISCUSSION}

\section{General topology of the phylogenetic tree}

A neighbour-joining tree based on partial $g y r B$ gene sequences $(1100 \mathrm{bp})$, representing $47 \%$ of the whole $g y r B$ gene sequence, was constructed (Fig. 2). The topology of the resulting tree was supported by the following observations. (i) The overall topology of the tree resembled that described for other sequenced and phylogenetically analysed genes of Helicobacter species, such as the 16S rRNA gene (Gueneau \& Loiseaux-De Goër, 2002), hsp60 (Mikkonen et al., 2004) and the 23S rRNA gene (Dewhirst et al., 2005). The tree contained three distinguishable clusters, wherein the first contained most of the gastric Helicobacter species (gastric cluster), the second was formed mainly by enterohepatic species (enterohepatic cluster) and the third included the species Helicobacter canadensis and Helicobacter pullorum. (ii) The maximum-parsimony tree based on the same sequences had a topology nearly identical to that of the neighbour-joining tree (results not shown). The only significant difference was seen in the positioning of Helicobacter mustelae, which had moved closer to the other gastric species, forming an 'intermediate species' between these two clusters. (iii) The mean bootstrap value 


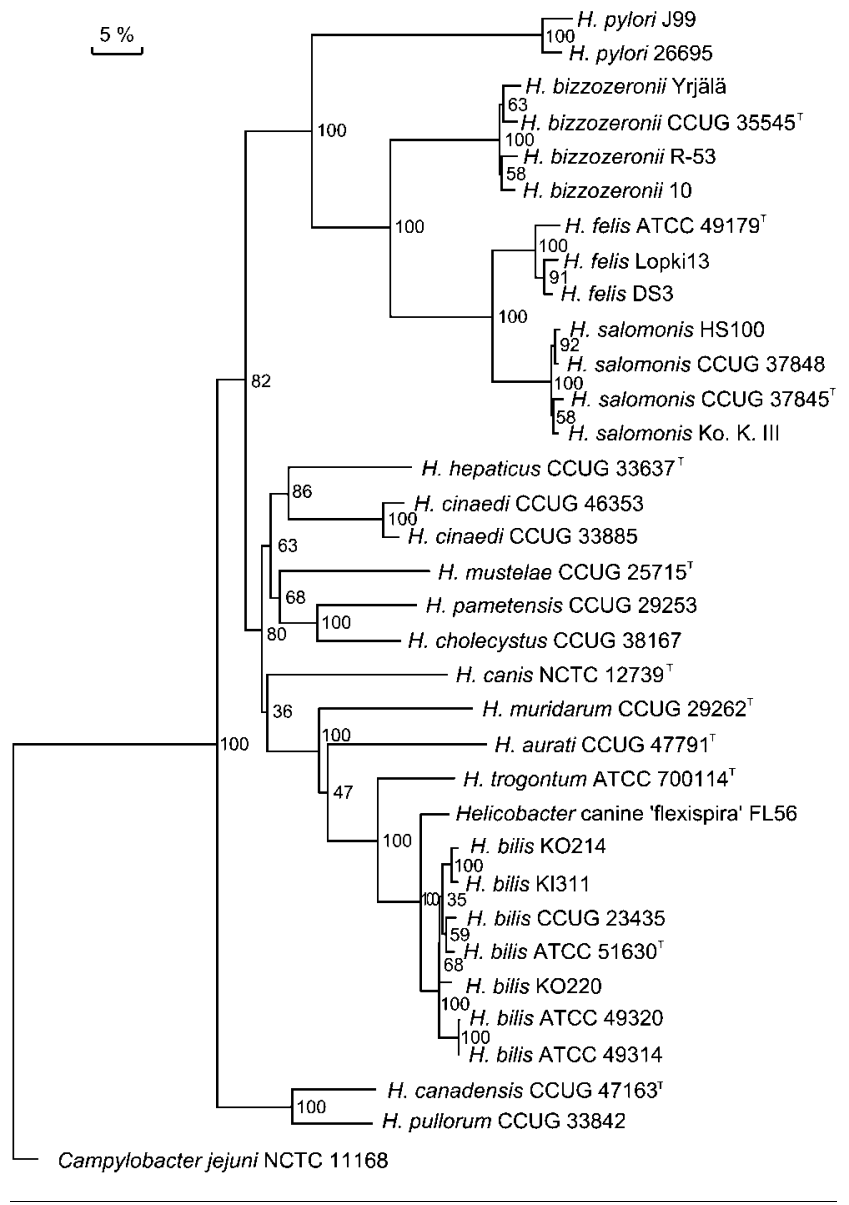

Fig. 2. Neighbour-joining tree based on partial gyrB gene sequences showing the phylogenetic relationships among members of the genus Helicobacter. The sequences were rooted with Campylobacter jejuni NCTC 11168. Numbers at nodes are percentage occurrence in 1000 bootstrapped trees. Bar, 5\% sequence divergence.

for all the nodes in the gyrB gene sequence neighbourjoining tree was $84 \%$, with only four bootstrap values under $60 \%$. The mean bootstrap value for the maximumparsimony tree was $74 \%$. Compared with the respective mean bootstrap values for the 16S rRNA gene sequence tree, 67 and $52 \%$ (Dewhirst et al., 2005), the phylogenetic trees based on the gyrB gene sequences appear to be far more robust. (iv) Introduction of different gap costs did not influence the resulting neighbour-joining tree.

The derived amino acid tree was similar to the DNA tree, but the branch lengths were considerably shorter as a consequence of most of the nucleic acid variation being caused by silent mutations in the third bases of the codons (results not shown).

Interspecies DNA similarity values of partial $\operatorname{gyr} B$ gene sequences ranged from $60 \%$ (between $H$. pullorum and $H$. felis) to $89 \%$ (between $H$. salomonis and $H$. felis). The lowest intraspecies similarity value, $94.6 \%$, was found among the two $H$. pylori strains tested.

The DNA G + C content of the fragments was $\leqslant 40 \mathrm{~mol} \%$ $(32.6-40.0 \mathrm{~mol} \%)$ in the enterohepatic species and $>46 \mathrm{~mol} \%$ (46.5-50.6 mol\%) in most of the gastric species, excluding $H$. pylori and $H$. mustelae, with $\mathrm{G}+\mathrm{C}$ contents of 34 and $42 \mathrm{~mol} \%$, respectively. The maximum difference between the lowest $\mathrm{G}+\mathrm{C}$ content (Helicobacter cholecystus) and the highest $\mathrm{G}+\mathrm{C}$ content (H. felis) was nearly $18 \mathrm{~mol} \%$, indicating high variability.

\section{Gastric species}

In the neighbour-joining tree, with the exception of $H$. mustelae, all the gastric Helicobacter species included in the study were grouped in the gastric cluster. Clustering of the gastric species was supported with high bootstrap values (100\%), indicating stable positions for these species (Fig. 2). The four H. bizzozeronii, three $H$. felis and four H. salomonis strains included in this cluster had intraspecies nucleotide similarity values of $96.2-96.9,95.6-98.0$ and $98.1-99.2 \%$, respectively, and interspecies similarity values of $74 \%$ between $H$. bizzozeronii and $H$. salomonis, $76 \%$ between H. bizzozeronii and $H$. felis and $89 \%$ between $H$. salomonis and $H$. felis. These results indicate high intraspecies similarity and a high interspecies difference, which is congruent with the speciation among this group. This distinction cannot be obtained based on 16S rRNA gene sequences because they generate similarity values between these three species that are as high as $99 \%$ (Jalava et al., 1997).

Although $H$. pylori clustered with the other gastric species, its sequence similarity to other Helicobacter species was low. The mean gyrB gene sequence similarity to the other gastric species was only $64 \%$ and to the enterohepatic species was $63 \%$. The DNA G $+\mathrm{C}$ content of the gyrB gene fragment in $H$. pylori was 8-17 mol\% lower than in other gastric species and about $5 \mathrm{~mol} \%$ lower than the whole-genome $\mathrm{G}+\mathrm{C}$ content of $H$. pylori (Alm et al., 1999). These results suggest that $H$. pylori differentiated a long time ago from animal gastric Helicobacter species and became adapted for the colonization of the human gastric area.

According to the partial gyrB gene sequences, H. mustelae clustered with the enterohepatic Helicobacter species and its gyrB DNA G + C content was $42 \mathrm{~mol} \%$, which is closer to the enterohepatic values ( $\leqslant 40 \mathrm{~mol} \%)$ than to the gastric values ( $>46 \mathrm{~mol} \%$ ). These results support the findings of Gueneau \& Loiseaux-De Goër (2002) and Mikkonen et al. (2004) and suggest that H. mustelae is an enterohepatic species by origin, but has relatively recently been able to acquire characteristics (e.g. the ureI gene) enabling it to alter its colonization niche from the intestinal to the gastric environment.

\section{Enterohepatic species}

The second cluster included most of the enterohepatic species. A tight subcluster was formed by the seven 
Helicobacter bilis strains, with 96.1-99.8\% intraspecies similarity at the nucleotide level. This further confirms the results of our previous study, in which phylogenetic analysis of fragments of ureB and $h s p 60$ gene sequences led us to conclude that the species $H$. bilis can be extended to include the reference strains of Helicobacter species 'flexispira' taxa 2, 3 and 8 (Hänninen et al., 2005). In the gyrB gene sequence tree all the $H$. bilis strains clustered tightly together and the other 'flexispira-like' Helicobacter species, $H$. trogontum, Helicobacter muridarum and Helicobacter aurati, were positioned in the vicinity of the H. bilis strains (Fig. 2). However, in the 16S rRNA gene sequence tree, the $H$. bilis strains were dispersed with weak bootstrap values within a cluster that also included the type strains of H. cinaedi, Helicobacter hepaticus and Helicobacter canis (Hänninen et al., 2005).

The canine strain FL57 is highly similar to H. bilis in terms of cell morphology and biochemical characteristics, but possesses 16S rRNA, hsp60, ureB (Hänninen et al., 2005) and, as shown in the present study, gyrB gene sequences that are distinct from the corresponding $H$. bilis sequences. Further studies are needed to establish whether this bacterium represents a novel Helicobacter taxon.

Another subcluster within the enterohepatic cluster was formed by $H$. mustelae, Helicobacter pametensis and $H$. cholecystus. There are few apparent characteristics that unify this subcluster, although similar clustering has been observed in 16S rRNA and 23S rRNA gene sequence trees (Dewhirst et al., 2005).

In the trees based on the ribosomal sequences, $H$. cinaedi strains are anomalously positioned among the taxa with flexispira morphology (Dewhirst et al., 2005). In the gyrB gene sequence tree, the $H$. cinaedi strains formed a separate subcluster clearly distinct from the species with 'flexispira' morphology.

The third cluster included the two helicobacters with unsheathed flagella, $H$. canadensis and $H$. pullorum. These species colonize the lower part of the gastrointestinal tract of birds. H. canadensis has also been isolated from pigs (Inglis et al., 2006). Their position in the tree was supported by a strong bootstrap value $(100 \%)$. The interspecies gyrB gene sequence similarity value between these two species was $85.1 \%$. The formation of a separate cluster for H. canadensis and $H$. pullorum can be explained by their sequence similarity to the other enterohepatic species, which was on average about $4 \%$ lower than within the enterohepatic species in general. The separate clustering of these species has also been observed in the $23 \mathrm{~S}$ rRNA gene sequence tree (Dewhirst et al., 2005).

\section{Development of species-specific PCR}

H. bizzozeronii is difficult to distinguish from $H$. felis and $H$. salomonis owing to high similarity in phenotypic and biochemical characteristics. Development of diagnostic methods for the detection of $H$. bizzozeronii, $H$. salomonis and $H$. felis in human gastric samples is now justified even more strongly following the publication of an investigation in which these species were identified, respectively, from 2.4, 11.4 and $8.9 \%$ of 123 human gastric biopsy specimens (Van den Bulck et al., 2005). These results indicate that these species, previously detected mainly from dogs and cats, also seem capable of infecting humans. The gyrB gene fragment analysis revealed differences in the nucleotide sequence of $H$. bizzozeronii relative to $H$. felis and $H$. salomonis that allowed us to develop a species-specific PCR method for the rapid diagnosis of $H$. bizzozeronii. As a result of PCR amplification using $H$. bizzozeronii-specific primers for several Helicobacter species and strains, only the four $H$. bizzozeronii strains produced a single band of $219 \mathrm{bp}$ in the electrophoresis gel.

In conclusion, the partial gyrB gene sequence analysis proved to be a particularly well-suited tool for phylogenetic studies. The overall topology of the phylogenetic tree based on the gyrB gene resembled that described for other sequenced and phylogenetically analysed genes of Helicobacter species, such as the $16 \mathrm{~S}$ rRNA gene (Gueneau \& Loiseaux-De Goër, 2002), hsp60 (Mikkonen et al., 2004) and the $23 S$ rRNA gene (Dewhirst et al., 2005). However, the gyrB gene seems to evolve at a much faster rate than, for example, the $16 \mathrm{~S}$ rRNA and $23 \mathrm{~S}$ rRNA genes, allowing the formation of clear interspecies differences at the sequence level (60-89\%) compared with the intraspecies similarity values (94.6-99.8\%). For example, the ribosomal sequences are unable to distinguish between certain Helicobacter species, such as $H$. felis and $H$. bizzozeronii, due to overlapping interspecies and intraspecies similarity values (Dewhirst et al., 2005). In addition, gyrB gene sequencing is straightforward as the sequences contain no intervening sequences and only a moderate number of primers are required, although consequently the amount of sequence information obtained $(\sim 1100 \mathrm{bp})$ is less than can be obtained with the more labour-intensive $23 \mathrm{~S}$ rRNA gene sequencing ( $\sim 2700 \mathrm{bp})$. It is therefore our recommendation, congruently with the multilocus sequence analysis approach suggested by Gevers et al. (2005), that studies on Helicobacter phylogeny should include the analysis of more than one established phylogenetic marker gene. Therefore, a reliable foundation for phylogenetic analysis of Helicobacter species could be based on joint data obtained from sequences such as the universally applied 16S rRNA gene and a suitable protein encoding gene, such as the $\operatorname{gyrB}$ gene.

\section{ACKNOWLEDGEMENTS}

This work was supported by a grant from the Academy of Finland. M. H. was funded by the ABS Graduate School. We are grateful to Urszula Hirvi for her technical assistance.

\section{REFERENCES}

Alm, R. A., Ling, L. S., Moir, D. T., King, B. L., Brown, E. D., Doig, P. C., Smith, D. R., Noonan, B., Guild, B. C. \& other authors (1999). 
Genomic-sequence comparison of two unrelated isolates of the human gastric pathogen Helicobacter pylori. Nature 397, 176-180.

Chenna, R., Sugawara, H., Koike, T., Lopez, R., Gibson, T. J., Higgins, D. G. \& Thompson, J. D. (2003). Multiple sequence alignment with the Clustal series of programs. Nucleic Acids Res 31, 3497-3500.

Dewhirst, F. E., Fox, J. G. \& On, S. L. (2000). Recommended minimal standards for describing new species of the genus Helicobacter. Int J Syst Evol Microbiol 50, 2231-2237.

Dewhirst, F. E., Shen, Z., Scimeca, M. S., Stokes, L. N., Boumenna, T., Chen, T., Paster, B. J. \& Fox, J. G. (2005). Discordant $16 \mathrm{~S}$ and $23 \mathrm{~S}$ rRNA gene phylogenies for the genus Helicobacter: implications for phylogenetic inference and systematics. J Bacteriol 187, 6106-6118.

Euzéby, J. P. (1997). List of Bacterial Names with Standing in Nomenclature: a folder available on the Internet. Int J Syst Bacteriol 47, 590-592. Last full update 4 April 2006. http://www.bacterio.cict.fr

Gevers, D., Cohan, F. M., Lawrence, J. G., Spratt, B. G., Coenye, T., Feil, E. J., Stackebrandt, E., Van de Peer, Y., Vandamme, P. \& other authors (2005). Opinion: re-evaluating prokaryotic species. Nat Rev Microbiol 3, 733-739.

Gueneau, P. \& Loiseaux-De Goër, S. (2002). Helicobacter: molecular phylogeny and the origin of gastric colonization in the genus. Infect Genet Evol 1, 215-223.

Hänninen, M. L., Happonen, I., Saari, S. \& Jalava, K. (1996). Culture and characteristics of Helicobacter bizzozeronii, a new canine gastric Helicobacter sp. Int J Syst Bacteriol 46, 160-166.

Hänninen, M. L., Utriainen, M., Happonen, I. \& Dewhirst, F. E. (2003). Helicobacter sp. flexispira $16 \mathrm{~S}$ rDNA taxa 1, 4 and 5 and Finnish porcine Helicobacter isolates are members of the species Helicobacter trogontum (taxon 6). Int J Syst Evol Microbiol 53, 425-433.

Hänninen, M. L., Kärenlampi, R. I., Koort, J. M., Mikkonen, T. \& Björkroth, K. J. (2005). Extension of the species Helicobacter bilis to include the reference strains of Helicobacter sp. flexispira taxa 2, 3 and 8 and Finnish canine and feline flexispira strains. Int J Syst Evol Microbiol 55, 891-898.

Inglis, G. D., McConville, M. \& de Jong, A. (2006). Atypical Helicobacter canadensis strains associated with swine. Appl Environ Microbiol 72, 4464-4471.

Jalava, K., Kaartinen, M., Utriainen, M., Happonen, I. \& Hänninen, M. L. (1997). Helicobacter salomonis sp. nov., a canine gastric Helicobacter sp. related to Helicobacter felis and Helicobacter bizzozeronii. Int J Syst Bacteriol 47, 975-982.

Jalava, K., On, S. L., Vandamme, P. A., Happonen, I., Sukura, A. \& Hänninen, M. L. (1998). Isolation and identification of Helicobacter spp. from canine and feline gastric mucosa. Appl Environ Microbiol 64, 3998-4006.

Jalava, K., On, S. L., Harrington, C. S., Andersen, L. P., Hänninen, M. L. \& Vandamme, P. (2001). A cultured strain of "Helicobacter heilmannii," a human gastric pathogen, identified as H. bizzozeronii: evidence for zoonotic potential of Helicobacter. Emerg Infect Dis 7, 1036-1038.

Kakinuma, K., Fukushima, M. \& Kawaguchi, R. (2003). Detection and identification of Escherichia coli, Shigella, and Salmonella by microarrays using the gyrB gene. Biotechnol Bioeng 83, 721-728.

Kasai, H., Ezaki, T. \& Harayama, S. (2000). Differentiation of phylogenetically related slowly growing mycobacteria by their gyrB sequences. J Clin Microbiol 38, 301-308.

Mikkonen, T. P., Kärenlampi, R. I. \& Hänninen, M. L. (2004). Phylogenetic analysis of gastric and enterohepatic Helicobacter species based on partial HSP60 gene sequences. Int J Syst Evol Microbiol 54, 753-758.

Paster, B. J., Lee, A., Fox, J. G., Dewhirst, F. E., Tordoff, L. A., Fraser, G. J., O'Rourke, J. L., Taylor, N. S. \& Ferrero, R. (1991). Phylogeny of Helicobacter felis sp. nov., Helicobacter mustelae, and related bacteria. Int J Syst Bacteriol 41, 31-38.

Pitcher, D. G., Saunders, N. A. \& Owen, R. J. (1989). Rapid extraction of bacterial genomic DNA with guanidium thiocyanate. Lett Appl Microbiol 8, 151-156.

Saitou, N. \& Nei, M. (1987). The neighbor-joining method: a new method for reconstructing phylogenetic trees. Mol Biol Evol 4, 406-425.

Sanders, M. K. \& Peura, D. A. (2002). Helicobacter pylori-associated diseases. Curr Gastroenterol Rep 4, 448-454.

Soler, L., Yanez, M. A., Chacon, M. R., Aguilera-Arreola, M. G., Catalan, V., Figueras, M. J. \& Martinez-Murcia, A. J. (2004). Phylogenetic analysis of the genus Aeromonas based on two housekeeping genes. Int J Syst Evol Microbiol 54, 1511-1519.

Van den Bulck, K., Decostere, A., Baele, M., Driessen, A., Debongnie, J. C., Burette, A., Stolte, M., Ducatelle, R. \& Haesebrouck, F. (2005). Identification of non-Helicobacter pylori spiral organisms in gastric samples from humans, dogs, and cats. J Clin Microbiol 43, 2256-2260.

Vandamme, P., Harrington, C. S., Jalava, K. \& On, S. L. (2000). Misidentifying helicobacters: the Helicobacter cinaedi example. J Clin Microbiol 38, 2261-2266.

Yamamoto, S. \& Harayama, S. (1995). PCR amplification and direct sequencing of $\operatorname{gyr} B$ genes with universal primers and their application to the detection and taxonomic analysis of Pseudomonas putida strains. Appl Environ Microbiol 61, 1104-1109. 